\title{
Dobutamine stress echocardiography for the detection of myocardial viability in patients with left ventricular dysfunction taking $\beta$ blockers: accuracy and optimal dose
}

\author{
T Zaglavara, R Haaverstad, B Cumberledge, T Irvine, H Karvounis, G Parharidis, \\ G Louridas, A Kenny
}

See end of article for authors' affiliations

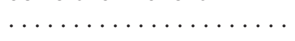

Correspondence to: Dr A Kenny, Department of Cardiology, Freeman Hospital, Newcastle upon Tyne NE7 7DN, UK;

antoinette.kenny@ncl.ac.uk

Accepted

26 November 2001

\begin{abstract}
Objective: To assess the accuracy of dobutamine stress echocardiography (DSE) and the optimal dose of dobutamine to detect myocardial viability in patients with ischaemic left ventricular (LV) dysfunction who are taking $\beta$ blockers, using the recovery of function six months artery revascularisation as the benchmark.

Patients: 17 patients with ischaemic LV dysfunction (ejection fraction $<40 \%$ ) and chronic treatment with $\beta$ blockers scheduled to undergo surgical revascularisation.

Setting: Regional cardiothoracic centre.

Methods: All patients underwent DSE one week before and resting echocardiography six months after revascularisation. A wall motion score was assigned to each segment for each dobutamine infusion stage, using the standard 16 segment model of the left ventricle. The accuracy of DSE to predict recovery of resting segmental function was calculated for low dose ( 5 and $10 \mu \mathrm{g} / \mathrm{kg} / \mathrm{min}$ ) and for a full protocol of dobutamine infusion ( 5 to $40 \mu \mathrm{g} / \mathrm{kg} / \mathrm{min}$ ).

Results: Of the 272 segments studied, 158 (58\%) were dysfunctional at rest, of which $79(50 \%)$ improved at DSE and $74(47 \%)$ recovered resting function after revascularisation. Analysis of results with a low dose showed a significantly lower sensitivity and negative predictive value than with a full protocol $(47 \% \vee 81 \%, p<0.001$ and $65 \% \vee 82 \%, p<0.05$, respectively). The accuracy in the full protocol analysis was comparable with that reported in patients no longer taking $\beta$ blockers but was significantly lower than that in the low dose analysis $(78 \% v 66 \%, p<0.001)$.

Conclusions: Findings suggest that $\beta$ blocker withdrawal is not necessary before DSE when viability is the clinical information in question. However, a completed protocol with continuous image recording is required to detect the full extent of viability.
\end{abstract}

$\mathrm{P}$ atients with ischaemic left ventricular (LV) dysfunction are increasingly referred for dobutamine stress echocardiography (DSE) studies for the assessment of myocardial viability. ${ }^{2}$ These patients usually have known significant coronary artery stenoses and are receiving antianginal treatment including $\beta$ blockers. There is convincing evidence from both experimental and clinical studies that $\beta$ blockers greatly attenuate the ability of DSE to induce ischaemia in the setting of a significant coronary lesion, due mainly to a blunted chronotropic effect. ${ }^{3-6}$ It has therefore been a common practice in most institutions to withdraw $\beta$ blockers for 3-5 half lives before performing a diagnostic DSE test.

$\beta$ Blocker withdrawal, however, can have deleterious effects in patients with LV dysfunction and significant coronary artery disease, increasing the risk of myocardial infarction, severe ventricular arrhythmias, and sudden death. ${ }^{7-10}$ While $\beta$ blocker withdrawal before myocardial viability studies does not appear to be either practical (gradual tapering over a period of several days to weeks $)^{71}$ or safe in this patient group, ${ }^{12}{ }^{13}$ there is limited information as to whether and how the accuracy of the test is affected by concurrent $\beta$ blockade.

The purpose of this study, therefore, was to assess the accuracy of DSE to detect myocardial viability in patients with ischaemic LV dysfunction who are taking $\beta$ blockers and to determine the optimal dose at which myocardial viability can best be detected.

\section{METHODS}

Patients scheduled to undergo coronary artery bypass grafting (CABG) at the Freeman Cardiothoracic Centre, Newcastle upon Tyne, UK, were enrolled between January 1999 and June 2000. The decision to use CABG was at the discretion of the referring cardiologist. Patients were included if they had significant two or three vessel disease, had impaired LV function with an ejection fraction $\leqslant 40 \%$ (measured by contrast or radionuclide ventriculography), and had been chronically treated with $\beta$ blockers. Exclusion criteria were (a) recent acute ischaemic event ( $<6$ weeks); (b) coexistent significant valve disease; (c) coronary anatomy unsuitable for complete revascularisation; (d) other surgical procedures scheduled in addition to CABG; (e) any contraindication to DSE; and (f) inadequate image quality, even after administration of ultrasound contrast agents. The hospital's ethics committee approved the study and informed consent was obtained from all patients.

All patients underwent DSE one week preoperatively and resting echocardiography six months after CABG.

Abbreviations: $C A B G$, coronary artery bypass grafting; $\mathrm{Cl}$, confidence interval; DSE, dobutamine stress echocardiography; HR; heart rate; LV, left ventricular; maxHR, maximum age predicted heart rate; WMSI, wall motion score index 


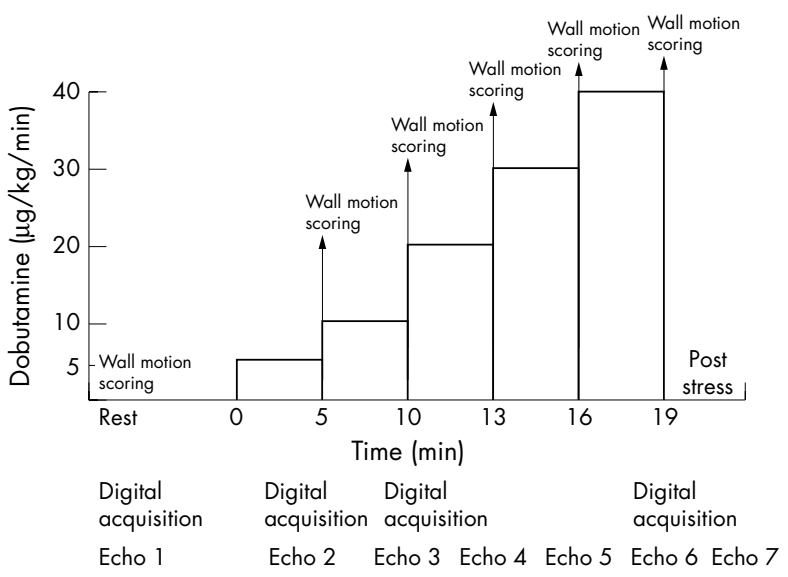

Figure 1 Dobutamine stress echocardiography protocol.

\section{Dobutamine infusion protocol}

Dobutamine was infused intravenously by means of an automated syringe pump starting at a dose of $5 \mu \mathrm{g} / \mathrm{kg} / \mathrm{min}$ for 5 minutes and increased to $10 \mu \mathrm{g} / \mathrm{kg} / \mathrm{min}$ for another 5 minutes. This was followed by infusions of 20,30 and $40 \mu \mathrm{g} / \mathrm{kg} / \mathrm{min}$ each lasting for 3 minutes (fig 1). Blood pressure and 12 lead ECG recordings were obtained at rest and at the end of each DSE stage. Reasons for termination of the infusion were severe hypertensive or hypotensive response, significant arrhythmias, prolonged angina, significant ECG changes, new wall motion abnormalities in at least two segments, achievement of $85 \%$ of the maximum age predicted heart rate (maxHR), and completion of the infusion protocol.

\section{Echocardiography}

An ATL HDI 5000CV system (Philips Medical Systems, Bothall, Washington, USA) was used and all studies were carried out using a single broadband transducer $(3 / 2 \mathrm{MHz})$ and tissue harmonic imaging. ${ }^{14}$ The standard parasternal and apical views were obtained at rest, at the end of each stage of dobutamine infusion, and at recovery and were stored on S-VHS videotape. Digital data were acquired on a quad screen format at rest and at the end of stages of 5, 10, and $40 \mu \mathrm{g} / \mathrm{kg} /$ min infusion using the digital stress acquisition package of the ATL HDI 5000CV (fig 1).

For reporting, the views obtained at 20 and $30 \mu \mathrm{g} / \mathrm{kg} / \mathrm{min}$ were displayed on a video player simultaneously with the quad screen display of the digitally acquired views at rest, with $5 \mu \mathrm{g} / \mathrm{kg} / \mathrm{min}$ and $10 \mu \mathrm{g} / \mathrm{kg} / \mathrm{min}$, and at peak. The standard 16 segment model proposed by the American Society of Echocardiography ${ }^{15}$ was used for wall motion analysis. All studies were interpreted in random order by a consensus of two experienced observers and each segment was scored for wall motion as $1=$ normal, $2=$ hypokinetic, and $3=$ akinetic. A wall motion score index (WMSI), calculated as the sum of individual segmental scores divided by 16, was assigned to each stage of the test. Evidence of wall thickening (score 1 or 2 ) in a previously akinetic segment (score 3 ) or normalisation of wall thickening (score 1) in a previously hypokinetic segment (score 2 ) was considered to be the criterion of myocardial viability in the preoperative DSE study. The same criteria were used to define recovery of resting segmental wall motion six months after CABG.

\section{Statistical analysis}

Continuous data are presented as mean (SD) and categorical data are expressed as proportions. The paired Student's $t$ test was used for comparison of continuous variables. The sensitivity, specificity, and positive and negative predictive values of DSE for the detection of myocardial viability were calculated by using the recovery of resting segmental wall
Table 1 Clinical, angiographic, and operative data of study patients $(n=18)$

\begin{tabular}{ll}
\hline & Number (\%) \\
\hline EF 25-40\% & $14(78)$ \\
EF <25\% & $4(22)$ \\
Previous myocardial infarction & $15(83)$ \\
Stable angina & $12(67)$ \\
Exertional dyspnoea & $11(61)$ \\
Q waves on ECG & $13(72)$ \\
Diabetes & $5(28)$ \\
Hypertension & $12(67)$ \\
Hyperlipidaemia & $13(72)$ \\
$\beta$ Blocker therapy & $18(100)$ \\
Calcium channel blocker therapy & $5(28)$ \\
Long acting nitrates & $6(33)$ \\
ACE inhibitors & $14(78)$ \\
Diuretics & $7(39)$ \\
Two vessel disease & $2(11)$ \\
Three vessel disease & $16(89)$ \\
Revascularisation vessels & \\
LIMA to LAD & $16(89)$ \\
LIMA to D1 & $2(11)$ \\
Radial to PDA & $1(6)$ \\
Radial to OMI & $2(11)$ \\
1 SVG & $2(11)$ \\
2 SVG & $5(28)$ \\
3 SVG & $9(50)$ \\
4 SVG & $2(11)$ \\
\hline
\end{tabular}

$A C E$, angiotensin converting enzyme; $D 1$, first diagonal artery; $E F$ ejection fraction; $L A D$, left anterior descending artery; LIMA, left internal mammary artery; $O M 1$, first obtuse marginal artery; PDA, posterior descending artery; SVG, saphenous vein graft.

motion six months after CABG as the benchmark. The $\chi^{2}$ test was used for comparison between proportions and the McNemar $\chi^{2}$ test for comparison between paired proportions. A probability value of $\mathrm{p}<0.05$ was considered significant.

\section{RESULTS}

\section{Patient characteristics}

Eighteen patients (16 male, 2 female, mean (SD) age 62 (9) years) were enrolled in the study. Ejection fraction was 34 (7) $\%$, range $17-40 \%$. At least one previous myocardial infarction was documented in 15 of the patients. Fifteen patients were taking atenolol 50-100 mg daily, two were taking metoprolol $50 \mathrm{mg}$ and $100 \mathrm{mg}$ daily, and one was taking bisoprolol $10 \mathrm{mg}$ daily. The patients' medications were not discontinued for the study and, in addition to $\beta$ blockers, six patients were taking nitrates, five were taking calcium channel blockers, seven were taking diuretics, and 14 were taking angiotensin converting enzyme inhibitors (table 1).

\section{Dobutamine stress echocardiography}

The full protocol was completed in 14 patients and was stopped at stage $4(30 \mu \mathrm{g} / \mathrm{kg} / \mathrm{min}$ ) in four (chest pain in two patients and new regional wall motion abnormalities in another two). Another two patients developed chest pain at the end of the peak stage $(40 \mu \mathrm{g} / \mathrm{kg} / \mathrm{min})$. No significant arrhythmias, hypotension, or other significant adverse events occurred. Figure 2 shows changes in heart rate (HR), blood pressure, and double product. HR was 56 (6) beats/min (35 (5)\% maxHR) at rest and increased to 83 (18) beats/min (53 (14)\% maxHR) at peak. HR at the state of maximal contractile response for each patient was 67 (14) beats/min (42 (9)\% maxHR), with an absolute increase of 13 (11) beats/min (range 0-30) over baseline.

One patient suffered a perioperative ischaemic event as documented by new wall motion abnormalities after CABG and was excluded from the analysis, which therefore included 17 patients. In total 272 myocardial segments were analysed ( 16 per patient), of which 158 (58\%) had baseline wall motion 


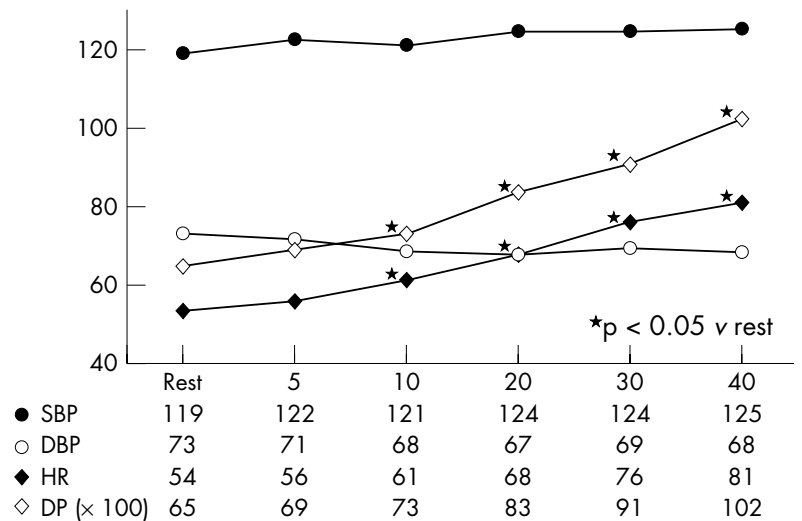

Figure 2 Line graph of changes in systolic blood pressure (SBP, $\mathrm{mm} \mathrm{Hg})$, diastolic blood pressure (DBP, $\mathrm{mm} \mathrm{Hg}$ ), heart rate (HR, beats $/ \mathrm{min}$ ), and double product (DP, $\mathrm{mm} \mathrm{Hg} \times$ beats $/ \mathrm{min}$ ) at rest and during each stage of dobutamine infusion $(\mu \mathrm{g} / \mathrm{kg} / \mathrm{min})$.

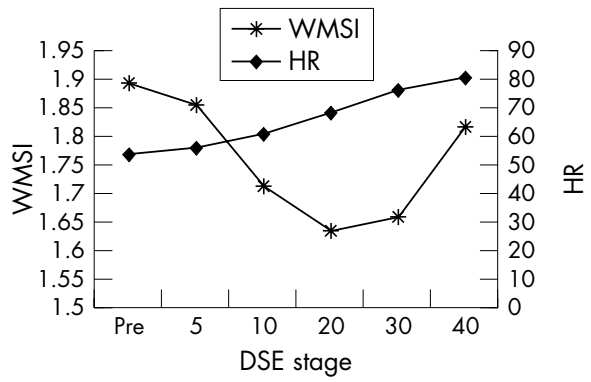

Figure 3 Line graph of the evolution of wall motion score index (WMSI) and heart rate (HR) during each stage of dobutamine stress echocardiography in patients taking $\beta$ blockers.

abnormalities ( 78 hypokinetic and 80 akinetic). The response of the 158 abnormal segments to dobutamine was as follows: continuous improvement was seen in 44 segments $(28 \%)$, biphasic response in $35(22 \%)$, ischaemic response in $4(3 \%)$, and no change in 75 segments $(47 \%)$. In addition, ischaemic response was shown in $9 \%$ ( 10 of 114) of the segments that were normal at baseline. Evidence of myocardial viability (continuous improvement or biphasic response) was shown in $26 \%$ ( 21 of 80 ) of the akinetic segments and in $74 \%$ ( 58 of 78 ) of the hypokinetic segments. Thus, myocardial viability was detected more frequently in hypokinetic than in akinetic segments $(\mathrm{p}<0.001)$.

Despite the blunted HR response, improvement in wall motion was evident from the end of stage 1 ( $5 \mu \mathrm{g} / \mathrm{kg} / \mathrm{min})$ with WMSI decreasing from $1.88(0.42)$ at rest to $1.84(0.43)$ $(\mathrm{p}<0.02)$. The WMSI decreased further to $1.70(0.43)$ at the end of stage $2(p<0.002)$ and to $1.62(0.45)$ at the end of stage $3(\mathrm{p}<0.002)$, and then increased significantly to $1.83(0.58)$ at the end of the peak stage ( $<<0.01$ ) (fig 3). Among 79 segments with improved wall motion, this improvement was evident at a low dose $(5-10 \mu \mathrm{g} / \mathrm{kg} / \mathrm{min})$ in $50(63 \%)$, at the end of $20 \mu \mathrm{g} / \mathrm{kg} /$ min in $18(23 \%)$, and at the end of $30 \mu \mathrm{g} / \mathrm{kg} / \mathrm{min}$ in $11(14 \%)$. Deterioration of function or deterioration as part of a biphasic response was not seen until the $30 \mu \mathrm{g} / \mathrm{kg} / \mathrm{min}$ stage ( 10 of 49 segments), but the majority of segments deteriorated at the peak stage of $40 \mu \mathrm{g} / \mathrm{kg} / \mathrm{min}$ (39 of 49 segments) (fig 4).

Patients were also analysed individually. Patients were classified according to the response observed in the majority of abnormal segments. A predominantly biphasic response was seen in four patients, continuous improvement in six, and no change in seven. Deterioration of function was not seen as the predominant response in any of the patients. At peak stress inducible ischaemia (new or worsening wall motion abnormalities, including biphasic responses) was evident in 11 of

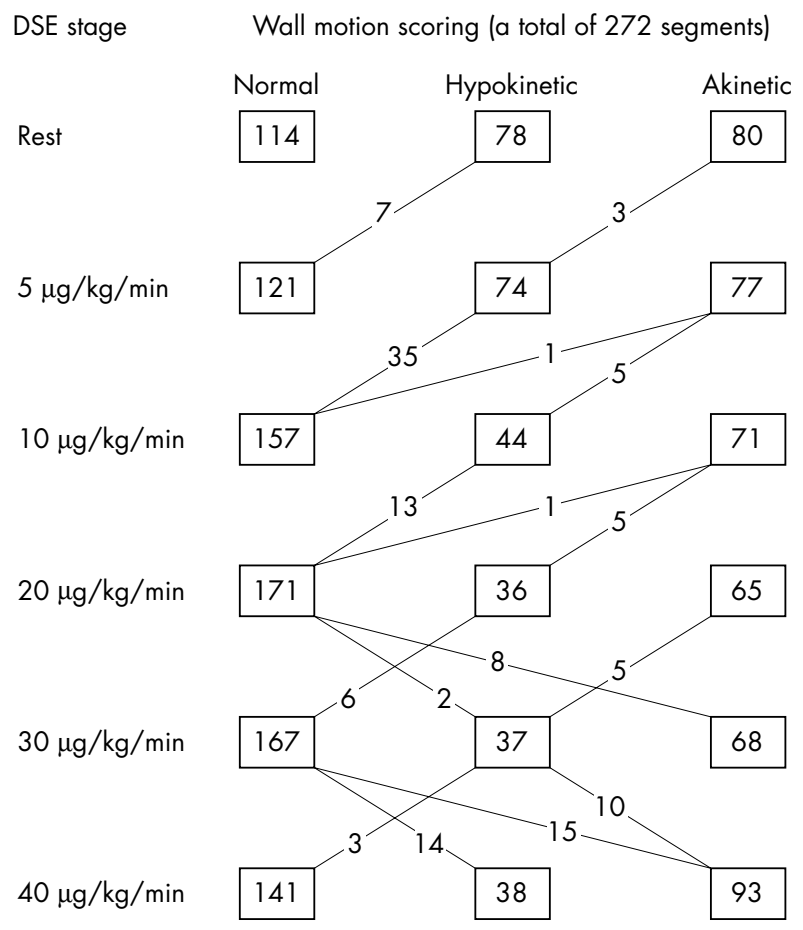

Figure 4 Chart of individual changes in segmental wall motion from baseline to each dobutamine infusion stage. DSE, dobutamine stress echocardiography.

the 17 patients (65\%). Despite the presence of significant coronary artery stenoses and completion of a full protocol (apart from one patient in whom the infusion was stopped at the end of $30 \mu \mathrm{g} / \mathrm{kg} / \mathrm{min}$ because of persistent chest pain), DSE failed to show any evidence of inducible ischaemia in 6 of the 17 patients $(35 \%)$.

\section{Coronary artery bypass grafting}

Every effort was made to complete the revascularisation during surgery and all epicardial vessels with significant stenoses were grafted. The mean (SD) number of distal coronary anastomoses was $3.6(0.8)$, range $2-5$ (table 1 ). Patients with anatomy unsuitable for complete revascularisation were not included in the study. Early graft function with intraoperative flow and resistance measurements was assessed in six patients using an ultrasonic transit time flow meter (MediStim AS, Oslo, Norway) and was found to be satisfactory. ${ }^{16}$

\section{Effect of coronary revascularisation on regional LV function}

None of the patients complained of angina six months after CABG. One patient had severe hypokinesis in the previously normal functioning lateral wall (three consecutive segments) and was therefore excluded from the analysis. In the remaining 17 patients, resting WMSI decreased to $1.58(0.44)$ from $1.88(0.42)$ preoperatively $(\mathrm{p}<0.001)$. The change in WMSI was significant in 12 patients $(p<0.05)$ and non-significant in five. Of the 158 dysfunctional segments, 74 (47\%) had improved function at the follow up study. In particular, improved function was evident in 26 of the 80 akinetic segments $(33 \%), 17$ of which improved to hypokinesis and nine to normal function. Of the 78 hypokinetic segments 48 $(62 \%)$ resumed normal function after revascularisation.

\section{Accuracy of dobutamine echocardiography in predicting recovery of segmental resting LV function six months after CABG}

In the 12 patients with significant improvement in resting WMSI, preoperative DSE showed an improvement in 5.9 (2.6) 
Table 2 Diagnostic accuracy of low dose and full protocol dobutamine echocardiography in predicting recovery of segmental resting function six months after revascularisation in patients taking $\beta$ blockers

\begin{tabular}{llllll}
\hline & Sensitivity (\%) & Specificity (\%) & PPV (\%) & NPV (\%) & Accuracy (\%) \\
\hline Low dose & $47(34 / 72)$ & $81(70 / 86)$ & $68(34 / 50)$ & $65(70 / 108)$ & $66(104 / 158)$ \\
$95 \% \mathrm{Cl}$ & 35 to 59 & 73 to 89 & 55 to 81 & 55 to 75 & 59 to 73 \\
Full protocol & $81(58 / 72)$ & $76(65 / 86)$ & $73(58 / 79)$ & $82(65 / 79)$ & $78(123 / 158)$ \\
$95 \% \mathrm{Cl}$ & 72 to 90 & 67 to 85 & 63 to 83 & 74 to 90 & 72 to 84 \\
p Value & $<0.001$ & NS & NS & $<0.05$ & $<0.001$
\end{tabular}

$\mathrm{Cl}$, confidence interval; NPV, negative predictive value PPV, positive predictive value.

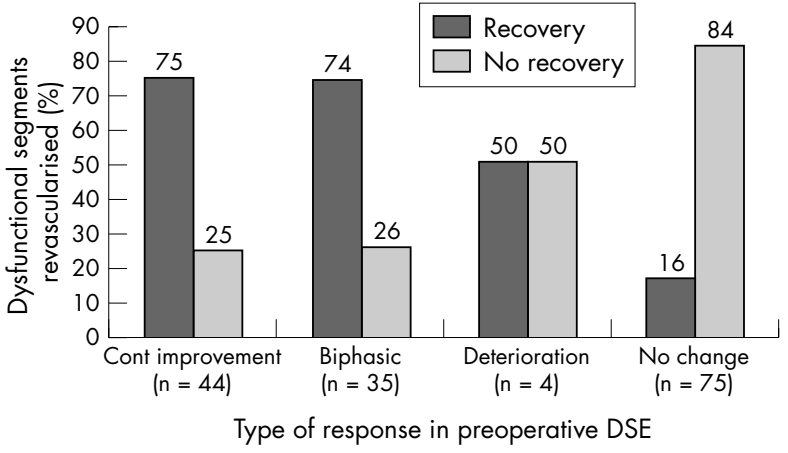

Figure 5 Bar graph showing percentage recovery of resting segmental left ventricular function six months after revascularisation analysed by the type of segmental wall motion response to preoperative dobutamine stress echocardiography (DSE).

segments per patient (range 3-12). In the five patients with no significant improvement in resting WMSI, preoperative DSE showed improvement in 1.6 (1) segments per patient (range $0-3)$. Of the 72 dysfunctional segments with improved function six months after CABG, $58(81 \%)$ had also improved during dobutamine infusion, whereas 65 of the 86 (76\%) segments with no recovery of function after CABG had not improved during DSE. Preoperative DSE had, therefore, correctly identified $81 \%$ of viable and $76 \%$ of non-viable segments (table 2 ).

Continuous improvement had a positive predictive value of $75 \%$ ( 33 of $44,95 \%$ confidence interval (CI) 0.62 to 0.88 ) for recovery of segmental resting function six months after CABG compared with $74 \%$ (26 of $35,95 \%$ CI 0.59 to 0.89 ) for biphasic response (NS). Deterioration of function in hypokinetic segments was rare (four segments), with a positive predictive value of $50 \%$ (two of four, 95\% CI 0.01 to 0.95 ) and was therefore not considered to predict viability in this study. Akinetic and dyskinetic segments were not evaluated for deterioration in function. Lack of any response to dobutamine had a negative predictive value of $84 \%$ ( 63 of $75,95 \%$ CI 0.76 to 0.92 ), whereas lack of any improvement with dobutamine had a negative predictive value of $82 \%$ ( 65 of $79,95 \%$ CI 0.74 to 0.90 ) (NS) (fig 5).
Improvement in akinetic segments during DSE had an excellent specificity ( 50 of $54,93 \%$ ) but a modest sensitivity ( 17 of $26,65 \%$ ) for recovery of resting function after CABG, whereas improvement in hypokinetic segments was more sensitive ( 41 of $46,89 \%, p<0.001$ ) but less specific ( 15 of 32 , $47 \%, \mathrm{p}<0.001)$. Overall accuracy was higher for akinetic than for hypokinetic segments (67 of $80(84 \%) \vee 56$ of $78(72 \%)$, $\mathrm{p}<0.1$ ) (table 3 ).

Compared with analysis of only the low dose ( 5 and $10 \mu \mathrm{g} /$ $\mathrm{kg} / \mathrm{min}$ ) results, analysis of all DSE stages had a better sensitivity (58 of $72(81 \%) v 34$ of $72(47 \%), p<0.001)$ and negative predictive value (65 of $79(82 \%) \vee 70$ of $108(65 \%)$, $\mathrm{p}<0.05)$. Analysis of all DSE stages had a slightly worse specificity than low dose analysis (65 of $86(76 \%) \vee 70$ of 86 $(81 \%))$ but this was not significant. The overall diagnostic accuracy of DSE to predict recovery of segmental wall motion six months after CABG was $78 \%$ ( 123 of 158 ) when the analysis considered all DSE stages and only 66\% (104 of 158) for the low dose analysis $(\mathrm{p}<0.001)$ (table 2$)$.

\section{DISCUSSION}

We have shown in a clinical setting of chronic hibernation and oral $\beta$ blockade that, during DSE, WMSI in the presence of $\beta$ blockade improves maximally at the end of the $20 \mu \mathrm{g} / \mathrm{kg} / \mathrm{min}$ infusion and not at the traditionally used low dose stages. The sensitivity of DSE to predict recovery of resting LV function after $C A B G$ is indeed low in patients taking $\beta$ blockers when analysis is based on the low dose protocol only. However, when analysis includes all infusion stages, DSE is both a sensitive and a specific predictor of the postoperative outcome of regional myocardial function in patients with ischaemic LV dysfunction who are taking $\beta$ blockers.

Because of their negative inotropic and chronotropic properties, $\beta$ blockers are expected to attenuate the physiological response to a given dose of dobutamine, ${ }^{3}{ }^{17}$ which acts mainly through selective $\beta 1$ adrenergic receptor stimulation. ${ }^{18}$ It has therefore been suggested that concurrent $\beta$ blockade may alter the ability of DSE to detect contractile reserve at low dose testing ${ }^{319}$ or modulate the response by causing less myocardial dysfunction for any given dose of dobutamine. ${ }^{20}$ In the era of antiadrenergic drugs as an established treatment for heart failure caused by ischaemic cardiomyopathy, ${ }^{21}$ the problem of this interaction becomes more clinically relevant, since

Table 3 Diagnostic accuracy of dobutamine echocardiography in predicting recovery of resting function for akinetic and hypokinetic segments six months after revascularisation in patients taking $\beta$ blockers

\begin{tabular}{llllll}
\hline & Sensitivity (\%) & Specificity (\%) & PPV (\%) & NPV (\%) & Accuracy (\%) \\
\hline Akinetic & $65(17 / 26)$ & $93(50 / 54)$ & $81(17 / 21)$ & $85(50 / 59)$ & $84(67 / 80)$ \\
$95 \% \mathrm{Cl}$ & 56 to 74 & 90 to 96 & 72 to 90 & 80 to 90 & 80 to 88 \\
Hypokinetic & $89(41 / 46)$ & $47(15 / 32)$ & $71(41 / 58)$ & $75(15 / 20)$ & $72(56 / 78)$ \\
$95 \% \mathrm{Cl}$ & 84 to 94 & 38 to 56 & 65 to 77 & 65 to 85 & 67 to 77 \\
p Value & $<0.001$ & $<0.001$ & NS & NS & $<0.1$ \\
\hline
\end{tabular}


patients with moderate to severe ischaemic LV dysfunction are usually taking $\beta$ blockers as a standard treatment. While $\beta$ blocker withdrawal is impractical and possibly dangerous in these patients, ${ }^{78}$ no practice guidelines or recommendations regarding this issue are available to clinicians performing myocardial viability studies.

Weissman and colleagues ${ }^{4}$ performed paired dobutamine infusion protocols with and without esmolol infusion in eight dogs with critical coronary artery stenosis. In their study the haemodynamic effects (including inotropic stimulation) at the low dose of dobutamine ( 5 and $10 \mu \mathrm{g} / \mathrm{kg} / \mathrm{min}$ ) were effectively eliminated in the presence of $\beta$ blockade, providing a rationale for the decreased sensitivity of low dose dobutamine to detect myocardial viability in patients taking $\beta$ blockers. In an experimental model of left anterior descending artery stenosis in pigs, Chen and colleagues ${ }^{5}$ showed that wall thickening in regions supplied by the stenotic left anterior descending artery had a typical biphasic response, increasing at lower doses of dobutamine and peaking at about $10 \mu \mathrm{g} / \mathrm{kg} / \mathrm{min}$ with a subsequent decrease at higher doses. In contrast, with esmolol infusion, regional wall thickening in the segments supplied by the stenotic left anterior descending artery was attenuated at rest and at the low dose of $5 \mu \mathrm{g} / \mathrm{kg} / \mathrm{min}$ compared with before esmolol infusion. With increasing doses of dobutamine, however, regional wall thickening increased continuously, with the maximal improvement at the dose of $20 \mu \mathrm{g} / \mathrm{kg} / \mathrm{min}$. Of interest, after the $10 \mu \mathrm{g} / \mathrm{kg} / \mathrm{min}$ stage wall thickening with esmolol significantly exceeded wall thickening without esmolol. In the same study Chen and colleagues ${ }^{5}$ further showed that $\beta$ blockade causes a shift between regional wall thickening and coronary flow per heart beat. Thus, with identical coronary flow reserves, there was greater wall thickening at peak dobutamine with esmolol than without it. Skopicki and associates ${ }^{22}$ have shown in humans with stable ischaemic heart disease that, in segments that function normally at baseline, an appropriate increase in regional contractile function with dobutamine infusion was facilitated rather than inhibited by $\beta$ blockers. As an explanation for this apparent paradox they suggest that the inotropic, chronotropic, and associated oxygen wasting properties of dobutamine may be sufficiently modulated by $\beta$ blockade to prevent ischaemia but not so completely blunted as to prevent an increase in regional contractility.

Preserved wall thickening at high doses of dobutamine despite the presence of a significant coronary stenosis is a major impediment to detecting inducible ischaemia. However, when viability and not ischaemia is the clinical information in question this may not be a problem, considering that early occurrence of ischaemia in areas with exhausted flow reserve may result in the inability to elicit enhanced contractile function despite the presence of myocardial viability. ${ }^{23}$

\section{Comparison with previous studies}

In a prospective cross over study of 26 patients, Fioretti and colleagues $^{6}$ found that $\beta$ blockers significantly reduced the incidence of clinical and echocardiographic markers of myocardial ischaemia during DSE. In the present study, as anticipated, DSE failed to show any evidence of inducible ischaemia in a significant proportion of the patients taking $\beta$ blockers $(35 \%)$, despite the presence of significant coronary artery disease. Ischaemic responses were rare (3\% of dysfunctional and $9 \%$ of normal segments) and deteriorated function was not seen as the predominant response in any of the patients.

In an analysis of pooled data from 16 studies published between 1980 and $1996,{ }^{24}$ low dose DSE (5-15 $\mu \mathrm{g} / \mathrm{kg} / \mathrm{min}$ ) was found to have a weighted mean sensitivity of $84 \%$ (range $71-97 \%$ ) and specificity of $81 \%$ (range $69-96 \%$ ) for the recovery of segmental resting function after revascularisation. In the majority of these studies, $\beta$ blockers were stopped for
48-72 hours ${ }^{25-28}$ before the DSE study. In some of the remaining studies either only a small proportion of the patients were taking $\beta$ blockers ${ }^{29}{ }^{30}$ or the patients' medications were not reported. ${ }^{31}$ In the present study the sensitivity and specificity of DSE in patients taking $\beta$ blockers were comparable with the above reported percentages ( $81 \%$ and $76 \%$, respectively) when analysis included all DSE stages. However, when analysis considered only the traditional "low dose" stages ( 5 and $10 \mu \mathrm{g} / \mathrm{kg}$ / $\mathrm{min}$ ) in assessing the improvement phase in wall motion, the sensitivity for detection of myocardial viability was extremely low. Afridi and colleagues ${ }^{30}$ in a study of 20 patients (of whom only eight were taking $\beta$ blockers) analysed wall motion scores for all DSE stages and concluded that doses of 5, 7.5, and $10 \mu \mathrm{g} / \mathrm{kg} / \mathrm{min}$ allowed detection of the improvement phase in all individual segments. Renewed deterioration in function during a biphasic response was seen as early as the $7.5 \mu \mathrm{g} / \mathrm{kg} /$ min dose, but the majority of segments deteriorated at a dose $\geqslant 20 \mu \mathrm{g} / \mathrm{kg} / \mathrm{min}$. In our study (including only patients taking $\beta$ blockers), the low dose (5 and $10 \mu \mathrm{g} / \mathrm{kg} / \mathrm{min}$ ) analysis allowed detection of the improvement phase in only $63 \%$ of the hibernating segments, while deterioration in function was not seen until the $30 \mu \mathrm{g} / \mathrm{kg} / \mathrm{min}$ dose with the majority of segments deteriorating at $40 \mu \mathrm{g} / \mathrm{kg} / \mathrm{min}$.

Several previous studies (with the majority of patients withdrawn from $\beta$ blockers) have established that the biphasic response to preoperative DSE most accurately predicts recovery of resting function after revascularisation, while continuous improvement has a very low positive predictive value..$^{29}{ }^{30}$ As an explanation for this finding, it has been suggested that chronic ischaemia is probably not the underlying mechanism of resting dysfunction in segments that improve continuously. Resting function is not therefore expected to improve in these segments with revascularisation. In our study of patients taking $\beta$ blockers, continuous improvement and biphasic response had similarly good positive predictive values (75\% and $74 \%$, respectively, NS) for the recovery of segmental wall motion six months after CABG. This is not surprising, however, considering that $\beta$ blockade is expected to prevent ischaemia in segments subtended by significantly stenosed vessels. Therefore, a biphasic response would have been seen in a significant number of segments with continuous improvement in the absence of $\beta$ blockade or in the presence of a cardiovascular stress higher than the peak dose dobutamine infusion, such as with administration of atropine. ${ }^{6}$

Myocardial viability was more common in hypokinetic than in akinetic segments and, in agreement with previous studies, ${ }^{27} 2832$ improvement in akinetic segments was extremely specific but modestly sensitive for functional recovery six months after CABG, while improvement in hypokinetic segments was much less specific but more sensitive. PerroneFilardi and colleagues, ${ }^{27}$ in a study of 18 patients in whom $\beta$ blockers had been withdrawn for 72 hours, reported that DSE had similarly high specificity and modest sensitivity (100\% and $69 \%$, respectively) for the recovery of function in akinetic segments. To explain the lower sensitivity of DSE in akinetic than in hypokinetic segments, it has been suggested that some akinetic segments may have exhausted coronary flow reserve and cannot respond to dobutamine with increased thickening despite the presence of myocardial viability. According to previously mentioned experimental data, ${ }^{5}$ for a given flow reserve $\beta$ blockade would be expected to facilitate wall thickening but this effect would be confined to high doses of dobutamine adequate to elicit ischaemia in the presence of exhausted flow reserve despite $\beta$ blockade. $\beta$ Blockade does not appear, therefore, either to compromise or to facilitate detection of myocardial viability in akinetic segments. Further studies comparing sensitivities in the same patients taking and withdrawn from $\beta$ blockers are needed to clarify this issue.

\section{Safety}

The safety of full dose dobutamine protocols in patients with known or suspected coronary artery disease has been assessed 
extensively in large scale studies and a low complication rate has been reported..$^{334}$ There is concern, however, that the incidence of severe complications may be higher in patients with myocardial ischaemia and severe LV dysfunction..$^{35}$ Several previous studies have used a full dose protocol in patients with hibernating myocardium to assess the diagnostic accuracy of the biphasic response, ${ }^{28-30} 36$ with no serious complications reported. Poldermans and colleagues ${ }^{37}$ studied 200 patients with ejection fraction $\leqslant 35 \%$, using a full dose dobutamine-atropine protocol, and reported a frequency of side effects similar to that in the pooled data from the literature. No correlation was found between the prevalence of cardiac arrhythmias and the extent of resting wall motion abnormalities or the induction of ischaemia. Elhendy and associates, ${ }^{38}$ however, reported an association between ventricular tachycardia during a dobutamine-atropine test and LV dysfunction. An interesting observation was that the prevalence of tachyarrhythmias requiring termination of the test was lower in the patients taking $\beta$ blockers.

In the present study no significant arrhythmias or other adverse effects occurred; however, atropine was not administered. In agreement with previous experimental data, ${ }^{4}$ the haemodynamic response during dobutamine infusion was blunted in patients taking $\beta$ blockers and the maximal cardiovascular stress achieved (expressed as HR and double product) at peak was significantly lower than that reported in patients withdrawn from $\beta$ blockers. ${ }^{6}{ }^{30}$ These energy sparing effects may translate into a better safety profile for patients taking $\beta$ blockers during dobutamine infusion similar to the cardioprotective effects seen with $\beta$ blockade during major vascular surgery. ${ }^{39}$ Obviously no conclusion can be reached on the basis of 17 patients and further studies with large numbers of patients are required to test this hypothesis.

\section{Study advantages and limitations}

To our knowledge this is the first study to assess the diagnostic accuracy of DSE for the detection of myocardial viability in patients with chronic ischaemic LV dysfunction taking $\beta$ blockers, using the recovery of resting function six months after CABG as the benchmark.

We studied only patients with at least moderate LV impairment so that, despite the relatively small number of patients, the number of dysfunctional myocardial segments was adequate to give significance to the results.

The patients in this study constitute a heterogeneous group with varying levels of $\beta$ blockade and underlying catecholamine stimulation. This is expected to affect the dose of dobutamine required for evidence of an inotropic or ischaemic response in each patient. While a full dose protocol with continuous image recording is required to detect the full extent of viability, this is not always possible from a clinical point of view. The mean absolute increase in HR over baseline at the state of the maximal inotropic response was 15 beats/min, ranging from $0-30$ beats $/ \mathrm{min}$. It can be assumed, therefore, that at an HR of 30 beats/min over baseline the dobutamine plasma concentrations are probably adequate to elicit the maximal contractile reserve in patients taking $\beta$ blockers. This can be used as an alternative to fixed dose analysis to guide dobutamine infusion when it is not possible to use a full protocol with continuous image recording.

Stress echocardiographic systems allow digital acquisition and simultaneous display of only four stages. Although the 20 and $30 \mu \mathrm{g} / \mathrm{kg} / \mathrm{min}$ stages were not acquired digitally, they were displayed on video simultaneously with the digitally acquired stages for reporting. As the maximal improvement in WMSI was seen at the end of the $20 \mu \mathrm{g} / \mathrm{kg} / \mathrm{min}$ stage, it is clinically important that this stage also be acquired digitally when software allowing acquisition of more than four stages becomes available.

The number of dysfunctional segments in which function deteriorated was too low in this study (four segments) and it is not possible to draw any conclusions about the accuracy of this type of response to predict viable myocardium. Increasing the dobutamine dose to more than $40 \mu \mathrm{g} / \mathrm{kg} / \mathrm{min}$ or administering atropine would probably have resulted in more ischaemic responses. This was not done, however, because the safety of these protocols has not been proved in this particular patient population.

Early graft failure may prevent functional recovery in otherwise viable segments, resulting in reduced specificity. Although angiography was not repeated six months after CABG to assess graft patency, early graft function was assessed intraoperatively in six patients as a quality control of the efficacy of revascularisation and was found to be satisfactory.

Ideally we should have repeated preoperative DSE in the same patients after withdrawing $\beta$ blockers for a head to head comparison of diagnostic accuracies. This was not done, however, because of patient safety considerations. Nevertheless, our findings that DSE has a high diagnostic accuracy to detect myocardial viability in patients taking $\beta$ blockers supports the argument that these agents need not be withdrawn before viability studies, given the increased risk of adverse events associated with $\beta$ blocker withdrawal in patients with ischaemic LV dysfunction.

\section{Conclusions and clinical implications}

Our findings suggest that $\beta$ blocker withdrawal is not necessary before DSE studies when viability and not ischaemia is the clinical information in question. However, some viability can be detected at higher doses than the traditional low dose stages and a completed protocol with continuous image recording is required to detect the full extent of viability. This protocol with infusion of high doses of dobutamine appears to be safe in this group of patients in whom $\beta$ blocker withdrawal is potentially dangerous; however, further studies with a large number of patients are required to confirm the safety of this protocol in patients with severe ischaemic LV dysfunction.

\section{ACKNOWLEDGEMENT}

This work has been supported with a research grant from the Medical Research Programme (Fourth Framework) of the European Commission.

\section{Authors' affiliations}

T Zaglavara, R Haaverstad, B Cumberledge, T Irvine, A Kenny, Cardiothoracic Centre, Freeman Hospital, Newcastle upon Tyne, UK H Karvounis, G Parharidis, G Louridas, Aristotle University, AHEPA Hospital, Thessaloniki, Greece

\section{REFERENCES}

1 Vanonerschelde JL, Pasquet A, Gerber B, et al. Pathophysiology of myocardial hibernation: implications for the use of dobutamine echocardiography to identify myocardial viability. Heart 1999;82(suppl III):IIII-7

2 Senior R, Lahiri A. Dobutamine echocardiography predicts functional outcome after revascularisation in patients with dysfunctional myocardium irrespective of the perfusion pattern on resting thallium-201 imaging. Heart 1999;82:668-73

3 Weissman NJ, Levangie WL, Newell JB, et al. Effect of $\beta$-adrenergic blockade on the physiologic response to dobutamine stress echocardiography. Am Heart J 1995;130:248-53.

4 Weissman NJ, Levangie WL, Guerrero JL, et al. Effect of $\beta$-blockade on dobutamine stress echocardiography. Am Heart J 1996;131:698-703.

5 Chen L, Ma L, De Prada V, et al. Effects of beta-blockade and atropine on ischemic responses in left ventricular regions subtending coronary stenosis during dobutamine stress echocardiography. J Am Coll Cardiol 1996;28: 1866-76.

6 Fioretti PM, Poldermans D, Salustri A, et al. Atropine increases the accuracy of dobutamine stress echocardiography in patients taking beta-blockers. Eur Heart J 1994; 15:355-60.

7 Eichhorn EJ. Beta-blocker withdrawal: the song of Orpheus. Am Heart J 1999;138:387-9.

8 Miller RR, Olson HG, Amsterdam EA, et al. Propranolol withdrawal rebound phenomenon: exacerbation of coronary events after abrupt cessation of antianginal therapy. N Engl J Med 1975;293:416-8.

9 Shand DG, Wood AJ. Propranolol withdrawal syndrome: why? Circulation 1978;58:202-3. 
10 Shammash JB Trost JC, Gold JM et al. Perioperative $\beta$-blocker withdrawal and mortality in vascular surgical patients. Am Heart $J$ $2001 ; 141: 148-53$

11 Prichard BN, Tomlison B, Walden RJ, et al. The beta-adrenergic blockade withdrawal phenomenon. J Cardiovasc Pharmacol 1983:5(suppl 1):S56-62.

12 Swedberg K, Hialmarson A, Waagstein F, et al. Adverse effects of beta-blockade withdrawal in patients with congestive cardiomyopathy. $\mathrm{Br}$ Heart J 1980;44:134-42

13 Ross PJ, Lewis $M$, Sheridan DJ, et al. Adrenergic hypersensitivity after beta-blocker withdrawal. Br Heart J 1981;45:637-42.

14 Zaglavara T, Norton $M$, Cumberledge $B$, et al. Dobutamine stress echocardiography: improved endocardial border definition and wall motion analysis with tissue harmonic imaging. J Am Soc Echocardiogr 1999; 12:706-13.

15 Schiller NB, Shah PM, Crawford M, et al. Recommendations for quantitation of the left ventricle by two-dimensional echocardiography. $J$ Am Soc Echocardiogr 1989;2:358-67.

16 Walpoth BH, Bosshard A, Genyk I, et al. Transit-time flow measurement for detection of early graft failure during myocardial revascularization. Ann Thorac Surg 1998;66:1097-100.

17 Everaert H, Vanhove C, Franken PR. Effect of beta-blockade on low-dose dobutamine-induced changes in left ventricular function in healthy volunteers: assessment by gated SPECT myocardial perfusion scintigraphy. Eur J Nucl Med 2000;27:419-24.

18 Opie L, Chatteriee K, Gersh BJ. Drugs for the heart. Orlando: Grune \& Straton, Inc, 1987:1,101.

19 Picano E. Echocardiographic recognition of myocardial viabiity. In: Stress echocardiography. Berlin: Springer-Verlag, 1997: 166-84

$20 \mathrm{Kaul} \mathrm{S}$. Response of dysfunctional myocardium to dobutamine: the eyes see what the mind knows. J Am Coll Cardiol 1996:27:1608-11.

21 Bristow M. $\beta$-adrenergic receptor blockade in chronic heart failure. Circulation 2000;101:558-69.

22 Skopicki HA, Abraham SA, Weissman NJ, et al. Factors influencing regional myocardial contractile response to inotropic stimulation: analysis in humans with stable ischemic heart disease. Circulation 1996:94:643-50.

23 Bonow RO. Identification of viable myocardium. Circulation 1996;94:2674-80

24 Bax JJ, Wijns W, Cornel JH, et al. Accuracy of currently available techniques for prediction of functional recovery after revascularization in patients with left ventricular dysfunction due to chronic coronary artery disease: comparison of pooled data. J Am Coll Cardiol 1997;30:1451-60.

25 La Canna G, Alfieri O, Giubbini R, et al. Echocardiography during infusion of dobutamine for identification of reversible dysfunction in patients with chronic coronary artery disease. J Am Coll Cardiol 1994;23:617-26.

26 Baer FM, Voth E, Deutsch HJ, et al. Predictive value of low dose dobutamine transesophageal echocardiography and fluorine-1 8 fluorodeoxyglucose positron emission tomography for recovery of regional left ventricular function after successful revascularization. J Am Coll Cardiol 1996;28:60-9.
27 Perrone-Filardi $P$, Pace L, Prastaro $M$, et al. Dobutamine echocardiography predicts improvement of hypoperfused dysfunctional myocardium after revascularization in patients with coronary artery disease. Circulation 1995;91:2556-65.

28 Vanoverschelde JL, D'Hondt AM, Marwick T, et al. Head-to-head comparison of exercise-redistribution-reinjection thallium single-photon emission computed tomography and low dose dobutamine echocardiography for prediction of reversibility of chronic left ventricular ischaemic dysfunction. J Am Coll Cardiol 1996;28:432-42

29 Qureshi U, Nagueh SF, Afridi I, et al. Dobutamine echocardiography and quantitative rest-redistribution $201 \mathrm{Tl}$ tomography in myocardial hibernation: relation of contractile reserve to $201 \mathrm{Tl}$ uptake and comparative prediction of recovery of function. Circulation 1997; 95:626-35.

30 Afridi I, Kleiman NS, Raizner AE, et al. Dobutamine echocardiography in myocardial hibernation: optimal dose and accuracy in predicting recovery of ventricular function after coronary angioplasty. Circulation 1995;91:663-70.

31 Haque T, Furukawa T, Takahashi $M$, et al. Identification of hibernating myocardium by dobutamine stress echocardiography: comparison with thallium-201 reinjection imaging. Am Heart J 1995;130:553-63.

32 Arnese M, Cornel JH, Salustri A, et al. Prediction of improvement of regional left ventricular function after surgical revascularization: a comparison of low-dose dobutamine echocardiography with $201 \mathrm{Tl}$ single-photon emission computed tomography. Circulation 1995;91:2748-52.

33 Secknus MA, Marwick TH. Evolution of dobutamine echocardiography protocols and indications: safety and side effects in 3,011 studies over 5 years. J Am Coll Cardiol 1997;29:1234-40.

34 Mathias W, Arruda A, Santos FC, et al. Safety of dobutamine-atropine stress echocardiography: a prospective experience of 4033 consecutive studies. J Am Soc Echocardiogr 1999;12:785-91.

35 Rahimtoola SH. Hibernating myocardium has reduced blood flow at rest that increases with low-dose dobutamine. Circulation 1996:94:3055-61.

36 Cornell JH, Bax JJ, Elhendy A, et al. Biphasic response to dobutamine predicts improvement of global left ventricular function after surgical revascularization in patients with stable coronary artery disease: implications of time course of recovery on diagnostic accuracy. J Am Coll Cardiol 1998;31:1002-10.

37 Poldermans D, Rambaldi R, Bax J, et al. Safety and utility of atropine addition during dobutamine stress echocardiography for the assessment of viable myocardium in patients with severe left ventricular dysfunction. Eur Heart J 1998;19:1712-8.

38 Elhendy A, van Domburg R, Bax J, et al. Relation between the extent of coronary artery disease and tachyarrhythmias during dobutamine stress echocardiography. Am J Cardiol 1999;83:832-5.

39 Poldermans D, Boersma E, Bax J, et al. The effect of bisoprolol on perioperative mortality and myocardial infarction in high-risk patients undergoing vascular surgery. N Engl J Med 1999;24:1789-94.

\section{IMAGES IN CARDIOLOGY}

\section{Anomalous left main stem stenosis}

A 82 year old man who was previously well was admitted to hospital with a five day history of unstable angina. His admission ECG was normal but subsequent ECGs showed $\mathrm{T}$ wave inversion in anterior leads. The peaked creatine kinase concentration was $555 \mathrm{u} / \mathrm{l}$. Despite medical treatment he had recurrent chest pain that was associated with dynamic inferolateral ST depression. Therefore, coronary angiography was performed. The left coronary artery could not be cannulated in the usual position. The right coronary artery was super dominant with an anomalous left coronary system arising at the ostium of the right coronary artery. The sole coronary stenosis was in the mid segment of the anomalous left main stem. Left ventricular function was preserved. He has been free of chest pain since his coronary artery bypass
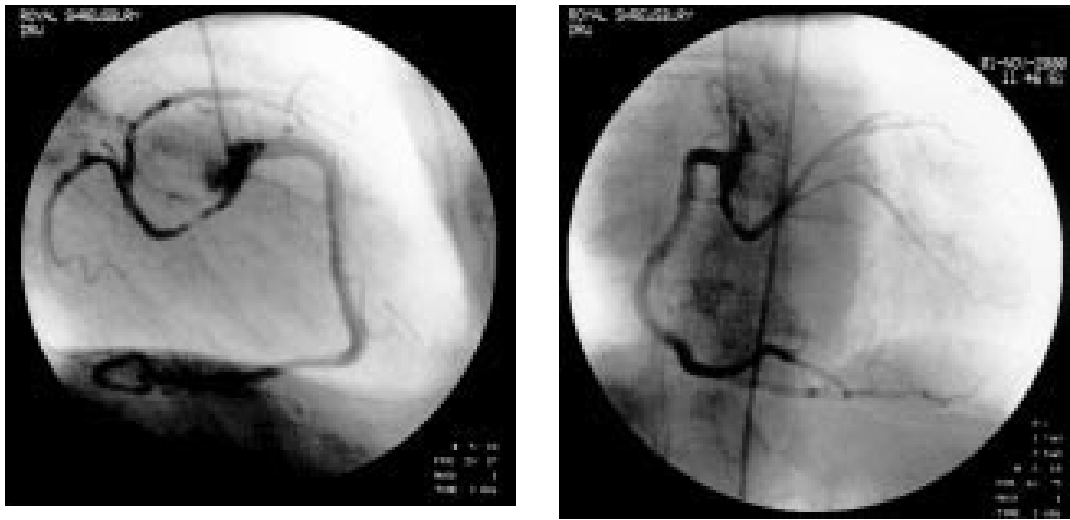
surgery.

T S N Lo 